\title{
Paraoxonase 1 decline and lipid peroxidation rise reflect a degree of brain atrophy and vascular impairment in dementia
}

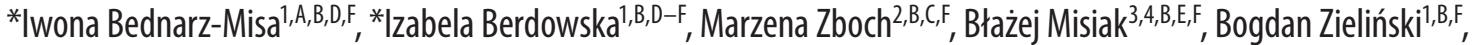 \\ Sylwia Płaczkowska ${ }^{5, B, F}$, Mariusz Fleszar ${ }^{1, B, F}$, Jerzy Wiśniewski ${ }^{1, B, F}$, Andrzej Gamian ${ }^{1, A, E, F}$, Małgorzata Krzystek-Korpacka ${ }^{1, A-F}$ \\ ${ }^{1}$ Department of Medical Biochemistry, Wroclaw Medical University, Poland \\ ${ }^{2}$ Alzheimer Center, Wroclaw Medical University, Ścinawa, Poland \\ ${ }^{3}$ Department of Psychiatry, Wroclaw Medical University, Poland \\ ${ }^{4}$ Department of Genetics, Wroclaw Medical University, Poland \\ ${ }^{5}$ Department of Professional Training in Clinical Chemistry, Wroclaw Medical University, Poland \\ A - research concept and design; B - collection and/or assembly of data; C - data analysis and interpretation; \\ $D$ - writing the article; $E$ - critical revision of the article; $F$ - final approval of the article
}

Address for correspondence

Izabela Berdowska

E-mail: izabela.berdowska@umed.wroc.pl

\section{Funding sources}

None declared

\section{Conflict of interest}

None declared

* Iwona Bednarz-Misa and Izabela Berdowska

contributed equally to this work.

Received on February 18, 2019

Reviewed on March 30, 2019

Accepted on July 28, 2019

Published online on January 22, 2020

Cite as

Bednarz-Misa I, Berdowska I, Zboch M, et al. Paraoxonase 1 decline and lipid peroxidation rise reflect a degree of brain atrophy and vascular impairment in dementia. Adv Clin Exp Med. 2020;29(1):71-78. doi:10.17219/acem/111377

DOI

10.17219/acem/111377

Copyright

Copyright by Author(s)

This is an article distributed under the terms of the

Creative Commons Attribution 3.0 Unported (CC BY 3.0)

(https://creativecommons.org/licenses/by/3.0/)

\begin{abstract}
Background. Paraoxonase 1 (PON1) is an enzyme with the capability to protect against lipid oxidation and atherosclerotic lesions formation. Impaired antioxidative capacity and enhanced lipid peroxidation (reflected by malondialdehyde rise) accompany dementias.

Objectives. The aim of this study was to discern the possible differences in the activity and phenotype distribution of PON1, and lipid peroxidation level in dementias of neurodegenerative and vascular pathology, to assess whether they reflect structural changes in the brain, and to evaluate their potential as dementia markers.

Material and methods. Paraoxonase 1 arylesterase activity and polymorphisms (dual-substrate method), and malondialdehyde/thiobarbituric acid reactive substances (MDA/TBARS) levels were determined spectrophotometrically in 257 serum samples derived from 136 dementive patients (with Alzheimer's disease $(A D ; n=63)$, vascular dementia (VaD; $n=40)$ and mixed-type dementia (MD; $n=33)$, as well as from 121 non-dementive individuals. The results were analyzed with reference to dementia type and severity (assessed with Mini Mental State Examination (MMSE) and Clinical Dementia Rating (CDR) scales), structural brain changes (estimated with magnetic resonance imaging (MRI) - Global Cortical Atrophy (GCA), Medial Temporal lobe Atrophy (MTA) and Fazekas scales)) and brain ischemia (Hachinski Ischemic Scale (HIS) index), and evaluated using receiver operating characteristic (ROC) analysis.
\end{abstract}

Results. Malondialdehyde/thiobarbituric acid reactive substances were increased in dementia (more in VaD than AD). In patients with vascular involvement, MDA/TBARS elevation reflected a degree of global cortical atrophy. Paraoxonase 1 activity was decreased in patients with dementia, especially in patients with severe cognitive deficits. In VaD, a drop in PON1 reflected a degree of MTA and brain ischemia. MDA/TBARS displayed $75 \%$ accuracy as a general dementia marker, but, similarly to PON1, were a poor differential marker.

Conclusions. Both indices were more associated with vascular involvement and the severity of brain atrophy or ischemia rather than with degree of cognitive decline.

Key words: Alzheimer's disease, vascular dementia, paraoxonase 1, mixed-type dementia, MDA/TBARS 


\section{Introduction}

Progressive degeneration of neurons, a hallmark of neurodegenerative disorders, leads to serious deficits in cognitive performance and general functioning. ${ }^{1}$ Due to the progressing aging of societies, the number of people suffering from various forms of dementia is projected to double every 20 years and will become a serious burden for public healthcare in the near future. Alzheimer's disease (AD) is the main cause of dementia in the elderly population, followed by vascular dementia (VaD). Alzheimer's disease is estimated to account for $60-80 \%$ of cases and is characterized by the accumulation of extracellular $\beta$-amyloid plaques and intracellular neurofibrillary tangles. ${ }^{1}$ Vascular dementia is the result of the blockage or damage of the cerebral blood vessels, leading to infarcts, bleeding and/or ischemia and ultimately to brain injury. It was initially considered to be the sole form of dementia attributable to vascular lesions; however, several studies have demonstrated that the development of cardiovascular risk factors also play a substantial role in AD. Indeed, neuropathological indices of brain infarcts can be found in up to half of AD patients. The co-existence of AD and VaD pathologies is observed in the vast majority of mixed dementia (MD) cases. ${ }^{1}$

Epidemiological studies have revealed that lipid peroxidation caused by oxidative imbalance is a common etiology of both cardiovascular ${ }^{2}$ and neurocognitive diseases. ${ }^{3}$ Among others, oxidative stress (OS) alters the permeability of vascular and cerebral endothelium and promotes inflammatory responses. ${ }^{3}$ Brain tissue is particularly vulnerable to OS due to high content of oxidative damage-prone polyunsaturated fatty acids and intense oxidative metabolism combined with low antioxidant capacity. ${ }^{3}$ Accordingly, the decline in antioxidants as well as the accumulation of oxidative damage markers, e.g., malondialdehyde (MDA), considered as the most abundant aldehyde derived from lipid peroxidation, have been reported in both $A D$ and $\mathrm{VaD} .^{3,4}$

Paraoxonase 1 (PON1) is a liver-synthesized enzyme of multiple biological functions, which has gained particular attention as an anti-atherogenic agent. It displays antioxidative properties and protects low-density lipoprotein (LDL) and high-density lipoprotein (HDL) lipids from oxidation, decreases oxidative status of macrophages and increases cholesterol efflux, thus contributing to the prevention and attenuation of the development of atherosclerotic lesions. ${ }^{5}$ Moreover, PON1 participates in the detoxification of homocysteine thiolactone, another pro-atherogenic compound, and plays an anti-inflammatory role by inhibiting the expression of monocyte chemoattractant protein (MCP)-1 and activity of myeloperoxidase, which serve as key players in vascular inflammation and OS. It has also been reported to stabilize lipid membranes and enhance their integrity under the conditions of oxidative imbalance. Accordingly, decreased enzyme activity has been repeatedly linked with an increased atherogenic risk. ${ }^{5}$
The study evaluated the potential relationship between oxidative imbalance and the degree of structural and functional impairment of the brain in patients with dementia of neurodegenerative and vascular pathology.

\section{Material and methods}

\section{Study population}

The study population consisted of 257 individuals: 136 with dementia and 121 without dementia who served as a control group. Among patients with dementia, 63 were diagnosed with $\mathrm{AD}, 33$ with MD and 40 with VaD. The following diagnostic criteria were used: 1$) \mathrm{DSM}^{-I^{6}}$ and NINCDS-ADRDA ${ }^{7}$ for AD; 2) ICD- $10^{8}$ and NINDS$\mathrm{AIREN}^{9}$ for $\mathrm{VaD}$, and 3) ICD-10 with the Hachinski Ischemic Scale (HIS) ${ }^{10}$ for MD. Patients with dementia were recruited from the Alzheimer Center, Wroclaw Medical University, Ścinawa, Poland (48 AD patients and all patients with MD and VaD) and from the Department of Psychiatry, Wroclaw Medical University (15 AD patients). All of the patients underwent a routine medical examination. Global cognitive function was assessed using the Mini Mental State Examination (MMSE) and Clinical Dementia Rating (CDR) scales. The presence of vascular involvement and global or focal atrophy were evaluated using magnetic resonance imaging (MRI) and estimated with the application of the following scales: the Global Cortical Atrophy (GCA) scale, the Medial Temporal lobe Atrophy (MTA) scale, and the Fazekas scale for white matter lesions. ${ }^{11}$ Computed tomography (CT) was used in patients in whom contraindications for MRI were observed (e.g., pacemaker or other metal elements in the body or fear of staying in confined spaces). The MRI scans were assessed by 2 trained persons (one of the authors (MZ) and an independent blinded radiologist), whose scores were averaged. Nutritional status of patients with dementia was evaluated using the body mass index (BMI) and the Mini Nutritional Assessment (MNA). ${ }^{12}$ Clinical characteristics of patients with dementia are presented in Table 1.

Control group consisted of the following:

1) BD group - 68 apparently healthy blood donors (age $>45$ years, no significant health history, no active inflammation, no pregnancy, no complaints concerning memory and cognitive function), recruited from the Regional Center for Blood Donation and Therapeutics in Wrocław, Poland;

2) HA group - 38 otherwise healthy individuals suffering from headaches, dizziness and/or complaining about weak memory in a degree justifying neuroimaging, but in whom neither loss of cognitive function nor any significant somatic or mental illnesses were diagnosed. They were recruited at the Alzheimer Center;

3) MCI group - 15 otherwise healthy individuals diagnosed with mild cognitive impairment (MCI) according 
Table 1. Characteristics of the study population

\begin{tabular}{|c|c|c|c|c|c|c|c|c|}
\hline \multirow{2}{*}{ Variables } & \multicolumn{4}{|c|}{ Patients with dementia (D) } & \multicolumn{3}{|c|}{ Not demented controls } & \multirow{2}{*}{$P_{\text {all }}$} \\
\hline & $\mathrm{AD}$ & MD & $\mathrm{VaD}$ & $P_{\text {dementia }}$ & $\mathrm{BD}$ & $\mathrm{HA}$ & $\mathrm{MCl}$ & \\
\hline Number of cases & 63 & 33 & 40 & & 68 & 38 & 15 & \\
\hline \multicolumn{9}{|c|}{ Demographics } \\
\hline Age [years] & $75.5 \pm 8.0$ & $75.6 \pm 7.0$ & $72.8 \pm 8.3$ & $0.185^{1}$ & $55.1 \pm 7.0$ & $61.6 \pm 8.8$ & $66.5 \pm 10$ & $<0.001^{1}$ \\
\hline Sex, F/M & $40 / 23$ & $22 / 11$ & $22 / 18$ & $0.551^{2}$ & $42 / 26$ & $28 / 10$ & $9 / 6$ & $0.559^{2}$ \\
\hline \multicolumn{9}{|c|}{ Nutritional status } \\
\hline BMI $\left[\mathrm{kg} / \mathrm{m}^{2}\right]$ & $27.1 \pm 4.1$ & $27.7 \pm 4.2$ & $28.7 \pm 5.4$ & $0.300^{1}$ & & $27.7 \pm 5.4$ & & $0.523^{1}$ \\
\hline MNA & $12.8 \pm 0.6$ & $12.9 \pm 0.4$ & $12.9 \pm 0.3$ & $0.811^{1}$ & & $13.8 \pm 0.7^{4}$ & & $<0.001^{1}$ \\
\hline \multicolumn{9}{|c|}{ Mental deficits } \\
\hline CDR & $1.48 \pm 0.58$ & $1.42 \pm 0.50$ & $1.48 \pm 0.55$ & $0.895^{1}$ & & $0^{4}$ & & $<0.0001^{3}$ \\
\hline MMSE & $17.5 \pm 4.2$ & $19.4 \pm 4.3$ & $18.7 \pm 4.0$ & $0.137^{1}$ & & $29.1 \pm 0.9^{4}$ & & $<0.001^{1}$ \\
\hline \multicolumn{9}{|c|}{ Neuroimaging and Hachinski Ischemic Scale } \\
\hline MTA & $2.5(2.4-3.0)$ & $2.5(2.0-3.5)$ & $2.63(2.3-3.0)$ & $0.725^{3}$ & & $0.5(0.0-0.5)^{4}$ & & $<0.0001^{3}$ \\
\hline GCA & $2.3 \pm 0.5$ & $2.3 \pm 0.5$ & $2.5 \pm 0.5$ & $0.133^{1}$ & & $0.9 \pm 0.5^{4}$ & & $<0.001^{1}$ \\
\hline Fazekas & $1.3(0.5-1.5)^{5,6}$ & $2(1.5-2.5)^{6,7}$ & $2.5(2-3)^{5,7}$ & $<0.00001^{3}$ & & $0.5(0.5-1.0)^{4}$ & & $<0.0001^{3}$ \\
\hline HIS & $3(2-3)^{5,6}$ & $5(5-6)^{6,7}$ & $7(7-8)^{5,7}$ & $<0.00001^{3}$ & & $2(1-3)^{4}$ & & $<0.0001^{3}$ \\
\hline
\end{tabular}

Data presented as means \pm standard deviation (SD) or medians with interquartile range. AD - Alzheimer's disease; MD - mixed-type dementia; VaD - vascular dementia; BD - healthy blood donors; HA - patients with headaches, dizziness and/or complaining about weak memory but without dementia or $\mathrm{MCl} ; \mathrm{MCl}$ - mild cognitive impairment; F/M - female-to-male ratio; BMI - body mass index; MNA - Mini Nutritional Assessment; CDR - Clinical Dementia Rating; MMSE - Mini Mental State Examination; MTA - Medial Temporal lobe Atrophy; GCA - Global Cortical Atrophy; HIS - Hachinski Ischemic Scale; ${ }^{1}$ - one-way analysis of variance (ANOVA); ${ }^{2}-X^{2}$ test; ${ }^{3}-$ Kruskal-Wallis $\mathrm{H}$ test; ${ }^{4}$ - significantly different from all remaining groups (AD, MD and VaD): ${ }^{5}$ - significantly different from $M D ;{ }^{6}$ - significantly different from $\mathrm{VaD} ;{ }^{7}$ - significantly different from $\mathrm{AD}$.

to Petersen's criteria ${ }^{13}$ were recruited from the Department of Psychiatry.

Basic demographic data of the control groups, including clinical characteristics of HA patients, are summarized in Table 1.

\section{Ethical considerations}

The study protocol was approved by the Medical Ethics Committee of Wroclaw Medical University (approval No. KB-679/2011 and KB-367/2017). The study was conducted in accordance with the Helsinki Declaration of 1975, as revised in 2013, and informed consent was obtained from all study participants. In the case of patients with severe dementia, legal guardians were consented.

\section{Analytical methods}

Blood samples were obtained following overnight fasting through venipuncture, clotted for $30 \mathrm{~min}$, and centrifuged $(15 \mathrm{~min}, 720 \times \mathrm{g})$. Serum was collected, aliquoted and kept frozen at $-80^{\circ} \mathrm{C}$ until examination.

\section{Malondialdehyde and malondialdehyde-like substances}

Serum concentrations of MDA/TBARS were determined spectrophotometrically with thiobarbituric acid (TBA) assay. ${ }^{14}$ To increase the specificity of the reaction, MDA/
TBARS were assessed in the presence of butylated hydroxytoluene (BHT) (Fluka Chemie, Buchs, Switzerland). ${ }^{15}$

\section{Measurement of PON1 activities and PON1 phenotyping}

To express PON1 activity, we determined its arylesterase activity against phenyl acetate as a substrate. Since phenyl acetate is equally metabolized by PON1 alloforms, which result from the most frequent Q192R polymorphism, the obtained enzymatic activity is considered as a surrogate for the enzyme concentration. ${ }^{16}$

To evaluate PON1 phenotype distribution, we used a dual substrate method, employing a determination of arylesterase and paraoxonase activities of the enzyme. ${ }^{17}$ Subsequent plotting of arylesterase against paraoxonase activity results in the separation of 3 forms of PON1, representing individuals homozygous for the $\mathrm{Q}$ alloenzyme (A phenotype of PON1 with Q at 192), individuals homozygous for the R alloenzyme (B phenotype of PON1 with R at 192), and heterozygous individuals ( $\mathrm{AB}$ phenotype of PON1). Due to the very low occurrence of $B$ phenotype, for the purpose of the present study, phenotypes $A B$ and $B$ were combined and are further described as phenotype $B$.

Paraoxonase 1 arylesterase and paraoxonase activities were determined spectrophotometrically by measuring the rates of hydrolysis of respective substrates: phenyl acetate (Sigma-Aldrich, St. Louis, USA) according to the Arylesterase/Paraoxonase assay kit protocol (ZeptoMetrix 
Co., Buffalo, USA) $(\mathrm{CV}=3.0 \%)$ and paraoxon (ChemService Inc., West Chester, USA) with the method designed by Charlton-Menys et al. ${ }^{18}$ One unit (U) of enzyme activity was defined as $1 \mathrm{mmol}$ of released phenol (arylesterase activity) or $1 \mu \mathrm{mol}$ of released p-nitrophenol (paraoxonase activity) per $1 \mathrm{~L}$ of serum per $1 \mathrm{~min}$. Intra-assay coefficients of variation (CV) for these methods were 1.1\% (paraoxonase activity) and 3\% (arylesterase activity). All measurements were conducted in duplicates and technical replicates were averaged.

\section{Statistical analysis}

Normality of data distribution was tested using the $\chi^{2}$ test and homogeneity of variances using Levene's test. Logtransformation was used, if appropriate. If not otherwise stated, data is presented as medians or means with $95 \%$ confidence interval $(95 \% \mathrm{CI})$. Continuous variables were analyzed using the Kruskal-Wallis $\mathrm{H}$ test or one-way analysis of variance (ANOVA) with Bonferroni correction for multiple testing and the t-test for independent samples with the Welch correction, if required. Correlation analysis was conducted using either the Spearman test $(\rho)$ or the Pearson's test (r). Frequency analysis and comparison of 2 proportions were conducted using the $x^{2}$ test. Ageand sex-adjusted analyses were conducted using the analysis of covariance (ANCOVA). The discriminative power of PON1 and MDA/TBARS was evaluated using the ROC analysis. Overall accuracy was expressed as an area under the ROC curve (AUC). Additionally, an optimal cut-off was determined and corresponding sensitivities and specificities were calculated. Backward method of multiple regression was used to discern independent predictors of PON1 activity and MDA/TBARS concentrations with $\mathrm{p}<0.05$ as inclusion and $\mathrm{p}>0.1$ as exclusion criteria. A two-tailed probability $<0.05$ was considered significant. The analyses were performed using MedCalc Statistical Software v. 17.4.4 (MedCalc Software bvba, Ostend, Belgium; https:// www.medcalc.org; 2017).

\section{Results}

\section{PON1 and MDA/TBARS in dementia}

Patients with dementia had significantly higher concentrations of MDA/TBARS and lower levels of arylesterase activity of PON1 (Fig. 1).

A detailed analysis revealed differences in MDA/TBARS in both control and dementia groups (Fig. 2). Among individuals recruited as controls, the $\mathrm{BD}$ group had significantly lower concentrations of MDA/TBARS than the HA or MCI group. Among patients with dementia, the presence of vascular impairment was responsible for significant upregulation of MDA/TBARS concentrations. Arylesterase activity of PON1 was significantly higher
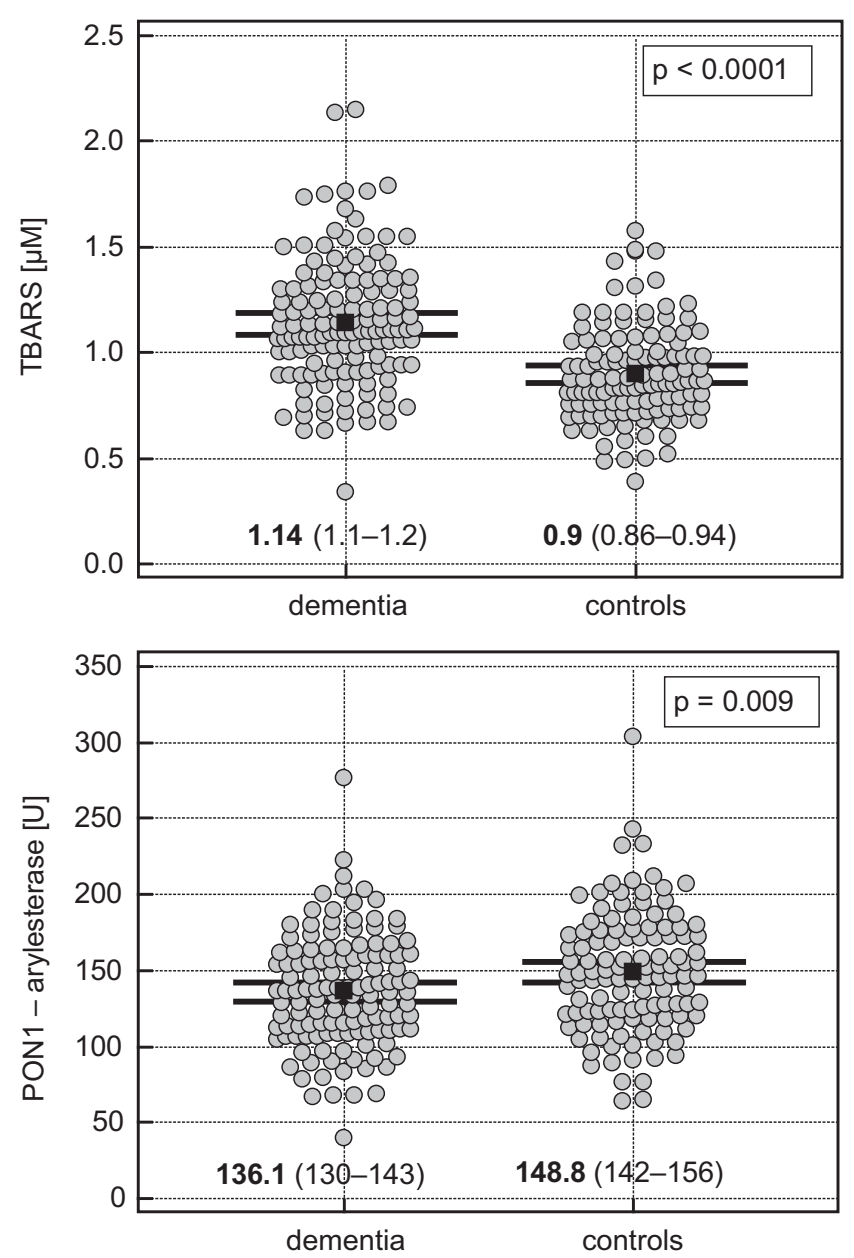

Fig. 1. Comparison of MDA/TBARS concentrations and PON1 activity between non-demented and demented individuals. Circles represent individual MDA/TBARS or PON1 values. Solid squares with whiskers represent means with 95\% confidence intervals (95\% Cl)

in $\mathrm{BD}$ as compared to other groups, with an exception of MCI, but did not show significant differences with respect to the type of dementia.

There was a significant age discrepancy between the control and dementia groups (Table 1). MDA/TBARS concentrations and $\mathrm{PON} 1$ arylesterase activities did not correlate with age in any of the studied groups (MDA/TBARS: $\mathrm{p}=0.790$ for $\mathrm{AD}$ group, $\mathrm{p}=0.405$ for $\mathrm{MD}$ group, $\mathrm{p}=0.526$ for $\mathrm{VaD}$ group, $\mathrm{p}=0.811$ for $\mathrm{BD}$ group, $\mathrm{p}=0.418$ for HA group, and $\mathrm{p}=0.720$ for MCI group; PON1: $\mathrm{p}=0.515$ for $\mathrm{AD}$ group, $\mathrm{p}=0.435$ for MD group, $\mathrm{p}=0.543$ for $\mathrm{VaD}$ group, $\mathrm{p}=0.935$ for $\mathrm{BD}$ group, $\mathrm{p}=0.498$ for HA group, and $\mathrm{p}=0.562$ for $\mathrm{MCI}$ group). However, there were significant correlations with age when the study population was analyzed as a whole $(r=0.43$, $\mathrm{p}<0.001$ for MDA/TBARS and $\mathrm{r}=-0.24, \mathrm{p}<0.001$ for PON1). Therefore, for the whole cohort evaluation, age- and sex-adjusted analysis was employed. Analysis of covariance revealed a significant effect of health status $(\mathrm{p}<0.001)$ and insignificant effects of age $(\mathrm{p}=0.931)$ and $\operatorname{sex}(\mathrm{p}=0.339)$ on MDA/TBARS. Similarly, health status ( $\mathrm{p}=0.041$ ), but not age $(\mathrm{p}=0.163)$ or $\operatorname{sex}(\mathrm{p}=0.071)$, was a significant predictor of PON1 arylesterase activity. 


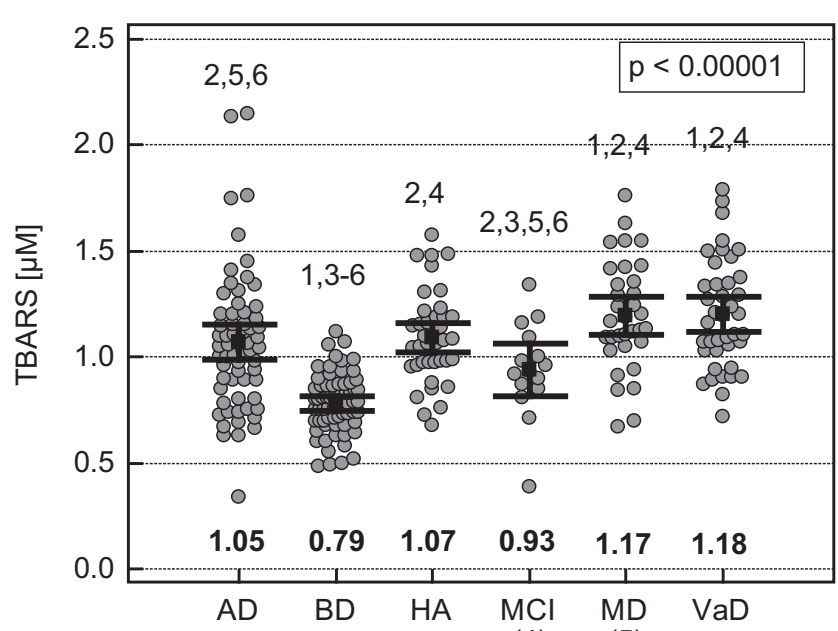

$\begin{array}{llllll}(1) & (2) & \text { (3) } & \text { (4) } & \text { (5) }\end{array}$

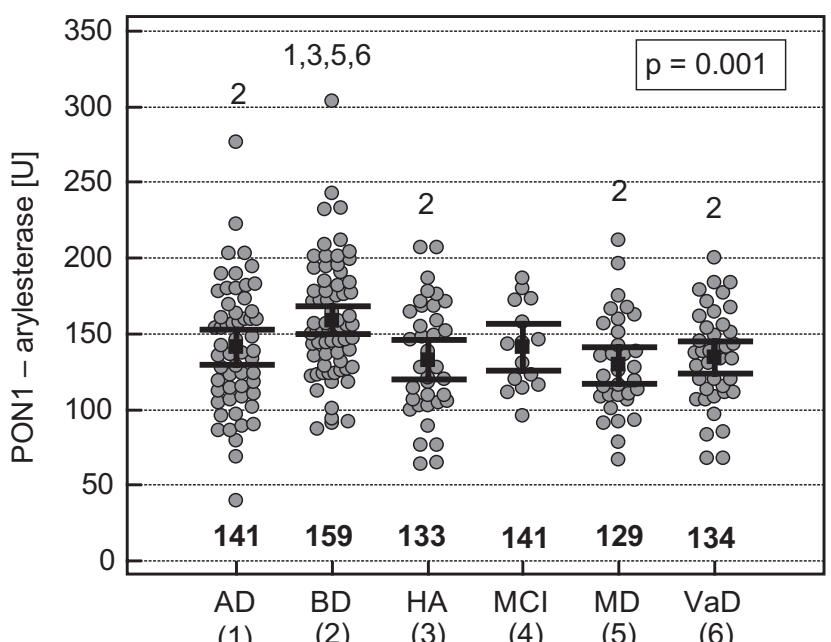

Fig. 2. Comparison of MDA/TBARS concentrations and PON1 activity between healthy individuals, individuals with benign headaches and memory deficits, patients with mild cognitive impairment, and patients with dementia of different pathology. Circles represent individual MDA/ TBARS or PON1 values. Solid squares with whiskers represent means with $95 \%$ confidence intervals $(95 \% \mathrm{Cl})$. The numbers above the data indicate significance of differences between groups in: Alzheimer's disease (1); blood donors (2); individuals with headaches, dizziness and/or complaining about weak memory but without dementia or $\mathrm{MCl}$ (3); mild cognitive impairment (4); mixed-type dementia (5); and vascular dementia (6)

\section{MDA/TBARS and PON1 as dementia and dementia differential markers}

The ROC analysis was employed to evaluate MDA/ TBARS and PON1 power in discriminating demented from non-demented individuals (dementia markers) as well as in differentiating dementia with and without vascular component (differential markers).

The AUCs for MDA/TBARS and PON1 as dementia markers were $0.75(\mathrm{p}<0.0001)$ and $0.59(\mathrm{p}=0.011)$, respectively. At the cut-off $>1.0 \mu \mathrm{M}$ and $\leq 142.2 \mathrm{U}$ for MDA/ TBARS and PON1, the sensitivities and specificities were as follows: $71 \%$ and $75 \%$ for MDA/TBARS and $60 \%$ and $59 \%$ for PON1.
The AUCs for MDA/TBARS and PON1 as differential markers were $0.58(\mathrm{p}=0.150)$ and $0.57(\mathrm{p}=0.269)$, respectively. At the cut-off $>1.061 \mu \mathrm{M}$ and $\leq 153.5 \mathrm{U}$ for MDA/ TBARS and PON1, the sensitivities and specificities were as follows: $73 \%$ and $46 \%$ for MDA/TBARS and $75 \%$ and $48 \%$ for PON1.

\section{PON1 phenotype distribution}

The prevalence of the A phenotype of PON1 was 63\% in $\mathrm{AD}$ group, $59 \%$ in $\mathrm{VaD}$ group, $50 \%$ in $\mathrm{MD}$ group, $47 \%$ in $\mathrm{MCI}$ group, $61 \%$ in $\mathrm{HA}$ group, and $48 \%$ in $\mathrm{BD}$ group. There was a $15 \%$ difference in the prevalence between $\mathrm{AD}$ and $\mathrm{BD}$ groups at the trend level significance $(\mathrm{p}=0.095)$.

In patients with vascular component (MD and $\mathrm{VaD}$ ) stratified by the presence of strategic infarcts, the prevalence of the A phenotype was $61.5 \%$ in patients without and $55.5 \%$ in patients with strategic infarcts $(\mathrm{p}=0.671)$.

\section{Interrelationship between PON1 alloforms and MDA/TBARS}

Controls with the phenotype A of the enzyme had significantly lower concentrations of MDA/TBARS than those with the phenotype $\mathrm{B}(0.85 \mu \mathrm{M}(0.8-0.9)$ vs $0.94 \mu \mathrm{M}$ (0.87-1.0), $\mathrm{p}=0.044)$. Similarly, MD patients with the phenotype A had significantly lower concentrations of MDA/ TBARS than those with the phenotype $B$ of the enzyme (1.1 $\mu \mathrm{M}$ (0.97-1.23) vs $1.29 \mu \mathrm{M}(1.15-1.42), \mathrm{p}=0.045)$. In the other studied groups, the phenotype-related difference in MDA/TBARS was not significant.

\section{The correlation of PON1 and MDA/TBARS with the degree of cognitive deficits}

The MDA/TBARS were positively correlated with MMSE in demented patients with vascular involvement (MD and VaD groups: $\rho=0.28, p=0.017$ ). Paraoxonase 1 did not exhibit any association with MMSE, but it correlated with CDR scale in the VaD group; the patients with CDR-1 grade had significantly higher PON1 activity than those with CDR-2 and CDR-3 grades (140.6 U (132-149) vs $127.4 \mathrm{U}(118-137), \mathrm{p}=0.040)$.

\section{PON1 and MDA/TBARS and the level of brain atrophy and vascular changes}

MDA/TBARS positively correlated with GCA scores in all patients who underwent neuroimaging $(\rho=0.21$, $\mathrm{p}=0.020, \mathrm{n}=127)$. The association was stronger in $\mathrm{pa}-$ tients with dementia and vascular involvement ( $\rho=0.30$, $\mathrm{p}=0.022, \mathrm{n}=57$ ), particularly those with strategic infarcts $(\rho=0.58, p=0.015, n=17)$. Arylesterase activity of PON1 negatively correlated with the MTA score in patients with dementia and with vascular involvement $(\rho=-0.27$, 
$\mathrm{p}=0.049, \mathrm{n}=57)$, particularly those with strategic infarcts $(\rho=-0.55, \mathrm{p}=0.024, \mathrm{n}=17)$. None of the parameters correlated with the Fazekas score.

\section{PON1 and MDA/TBARS and ischemia}

Arylesterase activity of PON1 negatively correlated with the HIS in VaD patients $(\rho=-0.46, \mathrm{p}=0.004)$ and in $\mathrm{pa}-$ tients with strategic infarcts $(\rho=-0.56, p=0.015)$. A similar tendency was observed in MD patients $(\rho=-0.34$, $\mathrm{p}=0.061)$. MDA/TBARS displayed only a tendency towards a weak correlation with the HIS score in a whole studied population $(\rho=0.14, p=0.073)$.

\section{Multivariate analysis}

The effect of mental deficits on MDA/TBARS and PON1 was co-examined with the effect of brain abnormalities detected with neuroimaging and with the HIS. The GCA score was the only variable retained in the multiple regression model explaining the MDA/TBARS variability with partial correlation coefficient $r=0.23$. The HIS was the only variable retained in the multiple regression model explaining PON1 variability with partial correlation coefficient $\mathrm{r}=-0.44$.

\section{Discussion}

In this study, we demonstrated an increase in MDA/ TBARS accompanied by a drop in PON1 activity in patients with dementia, particularly these with vascular involvement, which was related to the severity of brain atrophy or ischemia rather than to the degree of cognitive decline.

Oxidative stress has been shown to contribute to the pathogenesis of $\mathrm{AD}$ with the markers of oxidative imbalance evident both locally and systemically. ${ }^{19}$ Accordingly, the accumulation of MDA has been consistently reported in AD patients. ${ }^{4}$ There is a scarcity of studies comparing MDA levels between $\mathrm{AD}$ and $\mathrm{VaD}$ patients. In addition, these studies have yielded conflicting results, showing either equally high MDA levels in both types of dementia ${ }^{20}$ or significantly higher MDA accumulation in $\mathrm{AD}^{21}$ or, contrarily, in $\mathrm{VaD} .^{22}$ Taking into account difficulties in strict $\mathrm{AD}$ and $\mathrm{VaD}$ differentiation, a need for easily accessible biomarkers of vascular involvement has been stressed, and possible application of MDA has been suggested but not tested. ${ }^{22}$ In this study, we corroborated previous reports on elevated levels of MDA/TBARS in dementia. By showing MDA/TBARS to be significantly higher in $\mathrm{VaD} / \mathrm{MD}$ than $\mathrm{AD}$, we confirmed its particular association with vascular involvement. Our finding is in line with close cause-and-effect relationship between OS and vascular diseases supporting its involvement in the pathogenesis of $\mathrm{VaD} .{ }^{3}$ Adding to existing knowledge, we assessed MDA suitability as a differential dementia marker. However, despite its higher levels in dementia with vascular involvement, MDA/TBARS failed to display significant diagnostic power to be applied as a differential biomarker. Yet, MDA/ TBARS showed a moderate accuracy as a general dementia marker in our mixed cohort with AUC identical to previously reported ones for AD. ${ }^{23}$

Similarly to many other authors, ${ }^{24-28}$ we demonstrated a significant decrease in PON1 arylesterase activity in AD. However, studies comparing PON1 activity between distinct subtypes of dementia have yielded conflicting results. Bednarska-Makaruk et al. ${ }^{24}$ observed that a drop in PON1 activity was particularly expressed in the case of AD-related pathology, whereas Paragh et al. ${ }^{28}$ showed no differences. Others, like us, have found it to be lowered in dementias with vascular involvement. ${ }^{26,27,29}$ Moreover, Cervellati et al. ${ }^{30}$ reported low PON1 activity to be associated with an increased risk of $\mathrm{MCI}$ progression to $\mathrm{VaD}$ rather than AD. Even though the difference in PON1 activity between patients with dementia with and without vascular involvement in our cohort did not reach statistical significance, the enzyme association with vascular involvement seems to be confirmed by the negative correlation between PON1 activity and HIS index, reflecting the degree of ischemia. This finding is in agreement with the biological activity of PON $1^{5}$ and the results of epidemiological studies showing that the PON1 activity is inversely correlated with carotid and cerebral atherosclerosis. ${ }^{31}$

A direct correlation between MDA accumulation and cognitive decline is not unanimously observed. While some studies ${ }^{32}$ have reported erythrocyte and serum MDA to be inversely related to MMSE scores in AD patients, this relationship has not been mentioned by others. ${ }^{22,23,33}$ Liu et al. ${ }^{34}$ found this correlation in the general stroke patients but not in the subgroup suffering from post-stroke cognitive impairment. Similarly, the correlation between PON1 and MMSE has been reported only by 1 group of researchers. ${ }^{25} \mathrm{We}$, in turn, found MDA/TBARS to correlate with MMSE, but this association was counterintuitive, implying an improvement of cognitive function with the exacerbation of oxidative status. As expected, the association of PON1 with CDR suggested impaired protection against OS with the deterioration of cognitive functions. However, both correlations were weak and might have resulted from an interference with confounding factors. Indeed, when co-examined with the degree of brain alterations and HIS, the correlations between MDA/TBARS levels with MMSE, as well as between the PON1 activity and the CDR, lost their significance.

Quantitative structural MRI has evidenced structural changes in the brain already in the early phases of cognitive deterioration and the degree of regional atrophy has been associated with the severity of cognitive decline. Therefore, a more widespread application of neuroimaging in clinical routine practice, allowing for early and more accurate detection of dementia as well as its reliable 
differential diagnosis, has been postulated. ${ }^{11}$ The issue of possible correlation between biochemical markers of OS and neuroimaging has been addressed only recently. Bulboacă et al. ${ }^{35}$ demonstrated a positive correlation between markers of nitro-OS and posterior cortical atrophy. To our knowledge, neither MDA nor PON1 have been previously analyzed in the context of presence and the degree of brain alterations accompanying dementia. We found that in patients with dementia, particularly those with strategic infarcts (within VaD group), systemic MDA/TBARS accumulation was proportional to the degree of structural brain changes expressed in terms of GCA rating scale. Also, PON1 activity negatively correlated with the degree of medial temporal lobe atrophy (MTA rating scale). However, structural changes in the brain were independently associated only with MDA/TBARS, whereas they lost significance when their effect was co-evaluated with ischemia in the case of PON1.

Results from animal studies have shown OS to precede plaque formation characteristic for AD. ${ }^{36}$ The MCI is viewed as a transitional condition between physiological aging and $\mathrm{AD}$ and, as such, has gained increased attention. However, reports on OS in MCI are scarce and inconclusive. ${ }^{4}$ Although some authors have reported the lack of a significant difference in MDA between controls and MCI patients ${ }^{37}$ or its gradual increase along the sequence from healthy individuals to $\mathrm{MCI}$ and $\mathrm{AD},{ }^{32}$ we and others found serum MDA/TBARS to accumulate in MCI and AD patients to a similar extent, ${ }^{33}$ substantiating the notion that lipid peroxidation is a phenomenon that occurs early in the progression of dementia. ${ }^{34}$ Likewise, in the case of MDA/TBARS, there is no consensus in the literature concerning PON1 in MCI, the activity of which has been reported to resemble that in healthy controls ${ }^{29}$ or, contrarily, to be decreased similarly to patients with dementia. ${ }^{26,27,38}$ Also, our results are inconclusive - the PON1 activity in MCI was similar to that observed in AD; yet, MCI was the only group for which the difference, as compared to the healthy controls, did not reach statistical significance.

An interesting observation of our study was an evident oxidative imbalance in HA subgroup of controls comprising individuals admitted to the Alzheimer Center due to persistent headaches, dizziness and/or memory loss complaints. The etiology of their problems was not elucidated; neither the loss of cognitive function nor any significant somatic or mental illnesses were diagnosed. Nevertheless, these patients had significantly lower PON1 activity than $\mathrm{BD}$ controls recruited through the Blood Donation Center. Moreover, their concentration of lipid peroxidation markers (MDA/TBARS) exceeded that observed in both $\mathrm{BD}$ group and $\mathrm{MCI}$ patients. Therefore, our results implicate OS also in the pathogenesis of seemingly benign conditions.

Genetic studies addressing variations in the PON1 gene have revealed a lower frequency of the 192R allele among
AD patients than in the general population, suggesting its role as a protective factor against disease development. ${ }^{38}$ Although not consistently observed, this finding seems to corroborate our observations on the PON1 phenotype $\mathrm{B}$ that tended to be less prevalent in $\mathrm{AD}$ patients compared to healthy blood donors. The protecting effect attributed to the 192R allele might be associated with the PON1 activity as a natural inhibitor of cholinesterase. Cholinesterase inhibitors are employed as the first-line treatment for AD to prevent further loss of cognitive function via an increase in the availability of acetylcholine. ${ }^{39}$ On the other hand, the alloenzyme Q of PON1 is believed to be more efficient in counteracting lipid peroxidation. ${ }^{40}$ Accordingly, the concentrations of MDA/ TBARS were lower in the control group and MD patients with phenotype A of the enzyme at the trend level significance.

Our study has certain limitations that need to be discussed. Due to a natural history of neurocognitive disorders and the fact that the age over 65 years is considered a contraindication for blood donation, there was a significant difference in age distribution between controls and patients. However, the PON1 activity and MDA/TBARS levels correlated with age exclusively in the whole cohort and the issue was addressed in age- and sex-adjusted analysis. The ANCOVA confirmed that observed differences in the PON1 activity and MDA/TBARS levels could be attributed solely to a diagnosis of dementia and not to age or sex. The low number of patients with MCI as well as the lack of neuroimaging data or the assessment of degree of cognitive impairment is yet another limitation, negatively affecting the relevance of our findings concerning this particular group.

\section{Conclusions}

In this study, we confirmed an impaired oxidative status in patients with dementia and, for the first time, showed that an increase in MDA/TBARS level and a decrease in the PON1 activity reflects the severity of brain atrophy established with neuroimaging. We found ischemia, assessed with the application of HIS index, to be an independent predictor of the PON1 activity. Additionally, our findings suggest that OS also accompanies pathological changes in benign conditions.

\section{ORCID iDs}

Iwona Bednarz-Misa (D) https://orcid.org/0000-0001-7244-2017 Izabela Berdowska (D) https://orcid.org/0000-0002-0275-4522 Marzena Zboch (D) https://orcid.org/0000-0002-6853-0433 Błażej Misiak (D) https://orcid.org/0000-0002-5392-6398 Bogdan Zieliński (D) https://orcid.org/0000-0002-5330-9904 Sylwia Płaczkowska (D) https://orcid.org/0000-0002-1466-3820 Mariusz Fleszar (D) https://orcid.org/0000-0001-7857-327X Jerzy Wiśniewski (D) https://orcid.org/0000-0003-2831-7643 Andrzej Gamian (D) https://orcid.org/0000-0002-2206-6591 Małgorzata Krzystek-Korpacka (1) https://orcid.org/0000-0002-2753-8092 


\section{References}

1. Alzheimer's Association. 2016 Alzheimer's disease facts and figures. Alzheimers Dement. 2016;12(4):459-509.

2. Ahotupa M. Oxidized lipoprotein lipids and atherosclerosis. Free Radic Res. 2017;51(4):439-447. doi:10.1080/10715762.2017.1319944

3. Luca M, Luca A, Calandra C. The role of oxidative damage in the pathogenesis and progression of Alzheimer's disease and vascular dementia. Oxid Med Cell Longev. 2015;2015:504678. doi:10.1155/2015/504678

4. Schrag $M$, Mueller C, Zabel $M$, et al. Oxidative stress in blood in Alzheimer's disease and mild cognitive impairment: A meta-analysis. Neurobiol Dis. 2013;59:100-110. doi:10.1016/j.nbd.2013.07.005

5. Chistiakov DA, Melnichenko AA, Orekhov AN, Bobryshev YV. Paraoxonase and atherosclerosis-related cardiovascular diseases. Biochimie. 2017;132:19-27. doi:10.1016/j.biochi.2016.10.010

6. American Psychiatric Association. Diagnostic and Statistical Manual of Mental Disorders. $4^{\text {th }}$ ed. Washington, DC: American Psychiatric Association; 2000. doi:10.1002/jps.3080051129

7. McKhann G, Drachman D, Folstein M, Katzman R, Price D, Stadlan EM. Clinical diagnosis of Alzheimer's disease: Report of the NINCDSADRDA Work Group under the auspices of Department of Health and Human Services Task Force on Alzheimer's Disease. Neurology. 1984; 34(7):939-944. doi:10.1037/0894-4105.19.4.520

8. World Health Organization. International Statistical Classification of Diseases and Related Health Problems, $10^{\text {th }}$ Revision. Vol 41. Geneva, Switzerland: World Health Organization; 1992. http://www.who.int/ classifications/icd/ICD-10_2nd_ed_volume2.pdf.

9. Erkinjuntti T. Clinical criteria for vascular dementia: The NINDS-AIREN criteria. Dement Geriatr Cogn Disord. 1994;5(3-4):189-192. doi:10.1159/ 000106721

10. Hachinski V, Iliff L, Zilhka E, et al. Cerebral blood flow in dementia. Arch Neurol. 1975;32(9):632-637. doi:10.1001/archneur.1975.00490510 088009

11. Wahlund LO, Westman E, van Westen D, et al. Imaging biomarkers of dementia: Recommended visual rating scales with teaching cases. Insights Imaging. 2017;8(1):79-90. doi:10.1007/s13244-016-0521-6

12. Vellas B, Villars $H$, Abellan $G$, et al. Overview of the MNA: Its history and challenges. J Nutr Heal Aging. 2006;10(6):456-463.

13. Petersen RC. Mild cognitive impairment as a diagnostic entity. J Intern Med. 2004:256(3):183-194. doi:10.1111/j.1365-2796.2004.01388.x

14. Rice-Evans CA, Diplock AT, Symons MCR. Techniques in free radical research. In: Burdon RH, van Knippenberg PH, eds. Laboratory Techniques in Biochemistry and Molecular Biology. Vol. 22. Amsterdam, the Netherlands: Elsevier Science Publishers BV; 1991:1-291. doi:10.1016/S0075-7535(08)70046-9

15. Bartosz G. The Other Face of Oxygen. Free Radicals in the Environment [in Polish]. Warszawa, Poland: PWN; 2004.

16. Costa LG, Vitalone A, Cole TB, Furlong CE. Modulation of paraoxonase (PON1) activity. Biochem Pharmacol. 2005;69(4):541-550. doi:10. 1016/j.bcp.2004.08.027

17. La Du BN, Billecke S, Hsu C, Haley RW, Broomfield CA. Serum paraoxonase (PON1) isozymes: The quantitative analysis of isozymes affecting individual sensitivity to environmental chemicals. Drug Metab Dispos. 2001;29(4 Pt 2):566-569.

18. Charlton-Menys V, Liu Y, Durrington PN. Semiautomated method for determination of serum paraoxonase activity using paraoxon as substrate. Clin Chem. 2006;52(3):453-457. doi:10.1373/clinchem.2005. 063412

19. Massaad CA. Neuronal and vascular oxidative stress in Alzheimer's disease. Curr Neuropharmacol. 2011;9(4):662-673. doi:10.2174/1570 15911798376244

20. Polidori MC, Mattioli P, Aldred S, et al. Plasma antioxidant status, immunoglobulin $\mathrm{G}$ oxidation and lipid peroxidation in demented patients: Relevance to Alzheimer disease and vascular dementia. Dement Geriatr Cogn Disord. 2004;18(3-4):265-270. doi:10.1159/000080027

21. Casado A, Encarnación López-Fernández M, Concepción Casado M de La Torre R. Lipid peroxidation and antioxidant enzyme activities in vascular and Alzheimer dementias. Neurochem Res. 2008;33(3): 450-458. doi:10.1007/s11064-007-9453-3

22. Gustaw-Rothenberg K, Kowalczuk K, Stryjecka-Zimmer M. Lipids' peroxidation markers in Alzheimer's disease and vascular dementia. Geriatr Gerontol Int. 2010;10(2):161-166. doi:10.1111/j.1447-0594.2009. 00571.x
23. Lopez-Riquelme N, Alom-Poveda J, Viciano-Morote N, Llinares-Ibor I, Tormo-Diaz C. Apolipoprotein E \&4 allele and malondialdehyde level are independent risk factors for Alzheimer's disease. SAGE Open Med. 2016;4:2050312115626731. doi:10.1177/2050312115626731

24. Bednarska-Makaruk M, Graban A, Lipczyńska-Łojkowska W, et al. Positive correlation of paraoxonase 1 (PON1) activity with serum insulin level and HOMA-IR in dementia: A possible advantageous role of PON1 in dementia development. J Neurol Sci. 2013;324(1-2): 172-175. doi:10.1016/j.jns.2012.11.003

25. Wehr H, Bednarska-Makaruk M, Graban A, et al. Paraoxonase activity and dementia. J Neurol Sci. 2009;283(1-2):107-108. doi:10.1016/j.jns. 2009.02.317

26. Cervellati C, Romani A, Bergamini CM, et al. PON-1 and ferroxidase activities in older patients with mild cognitive impairment, late onset Alzheimer's disease or vascular dementia. Clin Chem Lab Med. 2015; 53(7):1049-1056. doi:10.1515/cclm-2014-0803

27. Castellazzi M, Trentini A, Romani A, et al. Decreased arylesterase activity of paraoxonase-1 (PON-1) might be a common denominator of neuroinflammatory and neurodegenerative diseases. Int J Biochem Cell Biol. 2016;81:356-363. doi:10.1016/j.biocel.2016.06.008

28. Paragh G, Balla P, Katona E, Seres I, Égerházi A, Degrell I. Serum paraoxonase activity changes in patients with Alzheimer's disease and vascular dementia. Eur Arch Psychiatry Clin Neurosci. 2002;252(2):63-67. doi:10.1007/s004060200013

29. Dantoine TF, Debord J, Merle L, Lacroix-Ramiandrisoa H, Bourzeix L, Charmes JP. Paraoxonase 1 activity: A new vascular marker of dementia? Ann N Y Acad Sci. 2002;977:96-101. doi:10.1111/j.1749-6632.2002. tb04802.x

30. Cervellati C, Trentini A, Romani A, et al. Serum paraoxonase and arylesterase activities of paraoxonase-1 (PON-1), mild cognitive impairment, and 2-year conversion to dementia: A pilot study. J Neurochem. 2015;135(2):395-401. doi:10.1111/jnc.13240

31. Arslan A, Tüzün FA, Arslan $\mathrm{H}$, et al. The relationship between serum paraoxonase levels and carotid atherosclerotic plaque formation in Alzheimer's patients. Neurol Neurochir Pol. 2016;50(6):403-409. doi:10.1016/j.pjnns.2016.07.002

32. Torres LL, Quaglio NB, De Souza GT, et al. Peripheral oxidative stress biomarkers in mild cognitive impairment and Alzheimer's disease. J Alzheimer's Dis. 2011;26(1):59-68. doi:10.3233/JAD-2011-110284

33. Balmus I-M, Strungaru S-A, Ciobica A, et al. Preliminary data on the interaction between some biometals and oxidative stress status in mild cognitive impairment and Alzheimer's disease patients. Oxid Med Cell Longev. 2017;2017:1-7. doi:10.1155/2017/7156928

34. Liu Z, Liu Y, Tu X, et al. High serum levels of malondialdehyde and 8-OHdG are both associated with early cognitive impairment in patients with acute ischaemic stroke. Sci Rep. 2017;7:9493. doi:10.1038/ s41598-017-09988-3

35. Bulboacă $A E$, Bulboacă SD, Bulboacă $A C$, Prodan Cl. Association between low thyroid-stimulating hormone, posterior cortical atrophy and nitro-oxidative stress in elderly patients with cognitive dysfunction. Arch Med Sci. 2017;13(5):1160-1167. doi:10.5114/aoms.2016. 60129

36. Praticò D, Uryu K, Leight S, Trojanoswki JQ, Lee VM-Y. Increased lipid peroxidation precedes amyloid plaque formation in an animal model of Alzheimer amyloidosis. J Neurosci. 2001;21(12):4183-4187. doi:21/ 12/4183 [pii]

37. Martín-Aragón S, Bermejo-Bescós P, Benedí J, et al. Metalloproteinase's activity and oxidative stress in mild cognitive impairment and Alzheimer's disease. Neurochem Res. 2009;34(2):373-378. doi:10.1007/ s11064-008-9789-3

38. Marsillach J, Parra S, Coll B, Joven J, Camps J. Paraoxonase-1 in chronic liver diseases, neurological diseases and HIV infection. In: Mackness B, Mackness M, Aviram M, Paragh G, eds. The Paraoxonases: Their Role in Disease Development and Xenobiotic Metabolism. Dordrecht, the Netherlands: Springer; 2008:187-198.

39. Wilkinson D, Francis P, Schwam E, Payne-Parrish J. Cholinesterase inhibitors used in the treatment of Alzheimer's disease. Drugs Aging. 2004;21(7):453-478. doi:10.2165/00002512-200421070-00004

40. Draganov DI, La Du BN. Pharmacogenetics of paraoxonases: A brief review. Naunyn Schmiedebergs Arch Pharmacol. 2004;369(1):78-88. doi:10.1007/s00210-003-0833-1 